\title{
CONDICIONANTES DEL DIAGNÓSTICO EN LA DEMANDA DE AYUDA PSICOLÓGICA EN UNA UNIDAD DE ATENCIÓN PSICOLÓGICA EN UN CAMPUS UNIVERSITARIO
}

\section{DETERMINANTS OF THE DIAGNOSIS IN THE DEMAND FOR PSYCHOLOGICAL AID IN A PSYCHOLOGICAL CARE UNIT IN A UNIVERSITY CAMPUS}

\author{
Alfred Tejedo García \\ Psicólogo Especialista en Psicología Clínica \\ Unitat d'Atenció Psicològica, Servei Assistencial de Salut \\ Universitat Autònoma de Barcelona, España \\ ORCID: https://orcid.org/0000-0002-9827-9059
}

Cómo referenciar este artículo/How to reference this article:

Tejedo García, A. (2019). Condicionantes del Diagnóstico en la Demanda de Ayuda Psicológica en una Unidad de Atención Psicológica en un Campus Universitario. Revista de Psicoterapia, 30(113), 213 237. https://doi.org/10.33898/rdp.v30i113.279

\begin{abstract}
Resumen
En este trabajo estudiamos las condiciones que correlacionan con el diagnóstico clínico que el psicólogo efectúa en las primeras visitas con los usuarios que consultan en la Unitat d'Atenció Psicològica del Servei Asssitencial de Salut de la Universitat Autònoma de Barcelona. Hacemos un estudio estadístico a partir de los historiales de los usuarios referidos al periodo 2010-2017, haciendo tablas de contingencia, $2 \times 2$, con 8 variables: edad, sexo, colectivo, primera consulta sobre el síntoma, tiempo de aparición de la sintomatología, motivo de la consulta, tratamiento psicofarmacológico y diagnóstico clínico. Nos centramos en la relación de dependencia de las variables con la variable principal, el diagnóstico. Resultados: Constatamos la relación de dependencia e interpretamos las interacciones significativas de todas las variables con la del diagnóstico, con la excepción de la edad y el sexo que son independientes. Discusión $y$ Conclusiones. Obtenemos un perfil de usuario propio del contexto universitario con diagnósticos prevalentes de trastorno adaptativo y en menor grado trastorno de ansiedad, en usuarios que mayoritariamente consultan por primera vez al psicólogo, que no están en tratamiento psicofarmacológico en el momento de la consulta, con una media de edad de 33 años, mayoritariamente mujeres, que aproximadamente la mitad consultan en intervalos de tiempo de menos de un año de tener síntomas. Lo que mueve a la consulta mayoritariamente tiene que ver con un sufrimiento mental menor respecto del sufrimiento que se encuentra en la red de salud mental pública.

Palabras clave: Diagnóstico, atención psicológica, universidad, estudio estadístico, variables.
\end{abstract}

Fecha de recepción: 19 de marzo de 2019. Fecha de aceptación: 22 de mayo de 2019.

Correspondencia sobre este artículo:

E-mail: Alfred.tejedo@uab.cat

Dirección postal: Av. de Can Domènech - Edifici F, 08193 Bellaterra, Barcelona. España

(C) 2019 Revista de Psicoterapia 


\begin{abstract}
In this work we study the conditions that correlate with the clinical diagnosis that the psychologist makes in the first visits with the users who consult in the Unitat d'Atencio Psicologica del Servei Asssitencial de Salut of the Universitat Autònoma de Barcelona. Method: We make a statistical study based on the records of the users referred to the period 2010-2017, making contingency tables, $2 \times 2$, with 8 variables: age, sex, collective, first consultation on the symptom, time of appearance of the symptomatology, reason for the consultation, psychopharmacological treatment and clinical diagnosis. We focus on the relationship of dependence of the variables with the main variable, diagnosis. Results: We found the relationship of dependence and interpreted the significant interactions of all the variables with the diagnosis, with the exception of age and sex that are independent. Discussion and Conclusions. We obtain a specific user profile of the university context with prevalent diagnoses of adaptive disorder and, to a lesser degree, anxiety disorder, in users who mostly consult the psychologist for the first time, who are not under psychopharmacological treatment at the time of consultation, with a average age of 33 years, mostly women, who approximately a half consult in time intervals less than a year of having symptoms. What moves the consultation mostly has to do with less mental suffering from the suffering that is found in the public mental health network.
\end{abstract}

Keywords: Diagnosis, psychological care, university, statistical study, variables. 


\section{Introducción}

En un trabajo previo ya nos referimos a las características de nuestra Unidad de Atención Psicológica de la Universitat Autònoma de Barcelona (Tejedo y López, 2009). Desde que se crearon entre 1984 y 1985, tanto los Servizos de Psicoloxía: Unidad de Tabaquismo y de "Venres Clínicos", en la Universidad de Santiago de Compostela, como la Unitat de Teràpia de Conducta de la Universitat de Barcelona, el número de servicios de asistencia psicológica a la población universitaria se ha ido incrementando. En 2009, nuestro unidad era uno de los 70 servicios existentes en 51 universidades del estado español, o más concretamente, uno de los 37 servicios de asistencia psicológica exclusivamente, es decir, que no ofrece atención psicopedagógica como sí lo hacen 11 servicios de esos 70 , ni una atención mixta, psicológica y psicopedagógica ${ }^{2}$, como lo efectúan otros 22 servicios entre los 70 (Saúl, López-González y Bermejo, 2009). Estos datos pueden haber cambiado pero desconocemos esa posible modificación. Nos lo hace pensar el dato de que disponemos a propósito de la existencia del número de servicios de atención psicológica, en 2004, 17 (García-Vera' ${ }^{2004)}$ y en 2008, 25 (Sánchez, Guillamón, Ferrer, Villalba, Martín y Pérez, 2008) ${ }^{3}$

Los datos de nuestra Unitat d'Atenció Psicològica (UAP) se han visto ligeramente modificados en los recursos humanos, respecto de los publicados en 2009. Todos los profesionales de la UAP seguimos siendo miembros externos a la comunidad universitaria de la Universidad Autónoma de Barcelona (UAB). Dos miembros del equipo somos psicólogos especialistas en psicología clínica, que hacemos el grueso de la jornada, mientras los otros dos, son psicólogos generales sanitarios ${ }^{4}$. Por otro lado, las horas de psiquiatría, si bien, han seguido existiendo, desde 2003 hasta 2018, en la actualidad, ya no se dispone de ellas, con lo cual, se ha vuelto a la situación inicial de la UAP, 1995, cuando no existía la figura del psiquiatra como figura prescriptiva de la indicación de asistencia psicológica, que se instauró en septiembre de 2003, aunque exclusivamente para los alumnos de la UAB sujetos al seguro escolar. Ha dejado de existir una prescripción basada en una normativa de 1953, es decir, preconstitucional, pero incomprensiblemente aún vigente en la legislación española (Tejedo, 2018).

A diferencia de muchos otros servicios universitarios del estado español compuestos, al menos parcialmente, por personal docente de la propia universidad (García-Vera, 2004), la condición de los profesionales de la UAP de ser externos a la comunidad universitaria, comporta que la totalidad de la jornada laboral está destinada a la asistencia clínica. La actividad docente también ha estado presente a lo largo de todos estos años ofreciendo prácticas a alumnos de Master de Psicoterapia Psicoanalítica de la Universitat de Girona, prácticamente hasta los años a los que se refiere el presente trabajo, a diferencia, también, de muchos otros servicios que ofrecen formación a los estudiantes de la propia universidad (Hernández y Froján, 1994).

La población a quienes se oferta la posible atención psicológica es, exclusiva- 
mente, a la población vinculada a la Universitat Autònoma de Barcelona, es decir, a cerca de 50000 personas, entre estudiantes, personal docente e investigador (PDI), becarios en formación y Personal de Administración y Servicios (PAS). Hay que resaltar que, a diferencia de algunos otros servicios, en la UAP los usuarios no tienen que pagar para ser atendidos, basta con ser miembro de la comunidad universitaria.

\section{Objetivo del estudio}

El propósito de este trabajo es revisar la casuística entorno a la primera visita con el psicólogo recibida en la Unitat d'Atenció Psicològica (UAP) del Servei Assistencial de Salut (UAP) de la Universitat Autònoma de Barcelona (UAB). Nos vamos a centrar en una muestra de 535 sujetos que han sido atendidos por los psicólogos del UAP pretendiendo hacer un trabajo reflexivo, intentando llegar a algunas hipótesis respecto de las condiciones del diagnóstico, a partir de los datos que disponemos referidos al período 2010-2017.

\section{Método}

En nuestro estudio partimos de los datos de la historia clínica del paciente y nos fijamos en una parte de la información tipificada y que es susceptible de categorización. Así, revisando cada caso de este período extenso de años de consultas, de un total de 1131 usuarios, excluimos los sujetos de los cuales faltan datos significativos, así como aquellos que no responden a intervención psicológica, es decir, a pacientes - muy escasos- que hubieran podido ser atendidos exclusivamente por psiquiatra ${ }^{5}$. Agrupamos la información a estudiar de manera estadística en 8 variables: edad, sexo, colectivo, primera consulta sobre el síntoma, tiempo de aparición de la sintomatología, motivo de la consulta, tratamiento psicofarmacológico, y diagnóstico clínico.

\section{Procedimiento}

El proceso de categorización de las variables supone una depuración de mucha información en unos casos más que en otros. Así, por ejemplo, mientras algunas variables no suponen mucho proceso de generalización, otras, sí; entre las primeras están: la edad, el sexo, el colectivo, existencia de tratamiento farmacológico o no, primera consulta sobre el síntoma, tiempo de aparición de la sintomatología ; entre las segundas: el motivo de la consulta y el diagnóstico clínico. El motivo de consulta supone un esfuerzo de síntesis de una dispersión de 21 categorías posibles, convertida en 7 variables. Hemos tenido que agrupar en una categoría "otros"motivos de consulta distintos (problemas sexuales, laborales, de sueño, de comportamiento, etc..) por razón de exigencia metodológica para trabajar los datos estadísticamente. El diagnóstico clínico una reducción de 40 diagnósticos distintos tipificados atendiendo a los criterios del Manual Diagnóstico y Estadístico de los trastornos mentales (4. ${ }^{\mathrm{a} E d}$.; DSM-IV; American Psychiatric Association [APA], 1994) DSMIV , agrupados en 10 variables, por la misma razón de exigencia estadística 
metodológica; en ellas agrupamos los distintos tipos de trastornos de ansiedad, de personalidad, de adaptación, depresivos, etc.

Las variables resultantes han sido las siguientes:

1) Edad del consultante. Categorizada en 5 intervalos: $<23$ años; $24-30$ años; 31-40 años; 41-50 años; > 51 años.

2) Sexo. Categorizada dicotómica: hombre o mujer.

3) Colectivo. Categorizada en 4 categorías: Estudiantes Universitarios; Becarios en formación; Personal no docente (PAS); Personal docente universitario (PDI).

4) Primera consulta. Variable categorizada en forma dicotómica: Sí, es la primera vez que consulta $(=1)$ No, ha consultado anteriormente $(=0)$.

5) Tiempo de aparición de la sintomatología. Categorizada en 5 categorías: < 1mes; 2-4 meses; 5-12 meses; de 13 a 60 meses; y > 61 meses.

6) Motivo de la consulta. Categorizada en 7 ítems: adaptación; ansiedad; estado de ánimo/autoestima; insatisfacción/inseguridad personal; problemas con la pareja; problemas relacionales (con la familia, con otras relaciones); otros.

7) Tratamiento farmacológico. Esta variable también está categorizada en forma dicotómica, sí/no: Sí, ha realizado tratamiento farmacológico anteriormente y recientemente $(=1)$. No, no ha realizado tratamiento farmacológico $(=0)$.

8) Diagnóstico clínico. Condensado en 10 categorías: trastornos adaptativos en general; trastornos adaptativos depresivos; trastornos adaptativos de ansiedad; trastornos de ansiedad en general; trastornos de crisis de ansiedad; trastornos fóbicos; trastornos depresivos; reacciones de duelo; trastornos de personalidad; otros trastornos (alimentación, tóxicos, sexualidad, psicosis, etc).

El estudio estadístico de los datos en que basamos nuestro trabajo lo hemos realizado a partir del soporte informático que supone el programa " $R$ ", en su versión 3.3.2, con su comando "Deducer", interfaz gráfica para R. Con los datos obtenidos realizamos estadística descriptiva e inferencial presentando los datos a partir de las tablas de contingencia que resultan del apareamiento de variables, dos a dos, y administrando pruebas de Chi Squared o de Likelihood ratio test, para valorar la relación de dependencia/independencia de las variables entre sí. Como se sabe, estas pruebas comparan los valores esperados (si la interacción es debida al azar) con los valores observados; $\mathrm{y}$ dan un valor ( $p$ valor) que es el que permite considerar que la relación de las variables es o no de dependencia $(p<0.05)^{6}$. 


\section{Resultados}

\section{A) Estudio estadístico}

\section{A.1. Análisis estadístico de variables}

Las frecuencias de las variables que identificamos de los 535 casos las consignamos a continuación, a saber:

1) Edad

Tabla 1. Edad.

\begin{tabular}{lll}
\hline Edad & Frecuencia & \% \\
\hline$<23$ & 95 & 17.8 \\
\hline $\mathbf{2 4 - 3 0}$ & $\mathbf{1 9 6}$ & $\mathbf{3 6 . 6}$ \\
\hline $\mathbf{3 1 - 4 0}$ & $\mathbf{1 2 8}$ & $\mathbf{2 3 . 9}$ \\
\hline $41-50$ & 56 & 10.5 \\
\hline$>51$ & 60 & 11.2 \\
\hline Total & 535 & 100 \\
\hline
\end{tabular}

La media de edad es de 32.86 años. La desviación estandard, 10.79 años. La mediana de edad, 30 años. El percentil 25, 25 años; y el percentil 75, 38 años. El límite mínimo, 18 años y el máximo, 67 años. Nuestros datos se acercan a los encontrados por Saldaña, Badós, García-Grau, balaguer (2009), 30.9 años, Labrador, Estupiñá y García-Vera (2010), 29.74 años (con límites mínimo y máximo 3 y 77, respectivamente), Calero, Torre Martí, Pardo y Santacreu (2018), media de 28.97 años (con límites mínimo y máximo 18 y 77, respectivamente) y en menor medida a los del estudio de Salaberría, Polo-López, Ruiz Iriondo, Cruz-Sáez y Echeburúa (2016) 25.71 años y a los de García-Vera (2004) 21-30 años, García-Vera y Sanz (2009) 18-25 años, Gutiérrez (2009), 47\% entre 18-30 años, Narváez y Aguirre (2016), menores de 30 años, y Tejedo y López (2009), 63.34\% entre 20-29 años.

2) Sexo

Tabla 2. Sexo.

\begin{tabular}{lll}
\hline Sexo & Frecuencia & $\mathbf{\%}$ \\
\hline Hombre & 175 & 32.7 \\
\hline Mujer & $\mathbf{3 6 0}$ & $\mathbf{6 7 . 3}$ \\
\hline Total & 535 & 100 \\
\hline
\end{tabular}

Estos datos también son muy próximos a los porcentajes de mujeres encontrados por Martorell y Carrasco (2009) 66.59\%, Labrador et al. (2010) 65.2\%, Calero et al. (2018) 64.7\%, Saldaña et al. (2009) 70\%, Avila, 
Herrero Sánchez y Fernández (2009) 70\% y, en parte, con los de Quesada (2004) entre $1 / 3$ de y $1 / 4$ de hombres, también en menor medida con los de García-Vera (2004) 71\% de mujeres, Tejedo y López (2009) 72.35\%, Salaberría et al. (2016) 75.6\% y García-Vera y Sanz (2009) 57.3\%. Y, alejados de los que refiere Narváez y Aguirre (2016) 48.9\%.

3) Colectivo

Tabla 3. Colectivo.

\begin{tabular}{lll}
\hline Colectivo & Frecuencia & $\mathbf{\%}$ \\
\hline Universitario & $\mathbf{3 5 5}$ & $\mathbf{6 6 . 4}$ \\
\hline Becario & 37 & 6.9 \\
\hline PAS & 104 & 19.4 \\
\hline PDI & 39 & 7.3 \\
\hline Total & 535 & 100 \\
\hline
\end{tabular}

Nuestros datos son significativamente diferentes de los que refiere Salaberría et al. (2016) $89.9 \%$ de alumnos, PAS 5\% y PDI $4.3 \%$ y en menor grado con nuestros propios datos de 2009, Tejedo y López (2009) 77.4\% alumnos, PAS $16.35 \%$ y PDI $6.25 \%$.

4) Primera consulta

Tabla 4. Primera consulta.

\begin{tabular}{lll}
\hline Primera consulta & Frecuencia & \% \\
\hline Sí & 374 & 69.9 \\
\hline No & $\mathbf{1 6 1}$ & $\mathbf{3 0 . 1}$ \\
\hline Total & 535 & 100 \\
\hline
\end{tabular}

Tanto Salaberría et al. (2016) como Labrador et al. (2010) encuentran unos resultados bastante diferentes: Para Salaberría et al. (2016), el 44.2\% ha realizado tratamiento anteriormente y Labrador (2010), el 49.3\% (frente al $30.1 \%$ de nuestro trabajo).

5) Tiempo de aparición de la sintomatología

Tabla 5. Tiempo de aparición de la sintomatología.

\begin{tabular}{lll}
\hline Tiempo & Frecuencia & \% \\
\hline$<1$ mes & 57 & 10.7 \\
\hline 2-4 meses & 122 & 22.8 \\
\hline $\mathbf{5 - 1 2}$ meses & $\mathbf{1 2 4}$ & $\mathbf{2 3 . 2}$ \\
\hline $\mathbf{1 3 - 6 0}$ meses & $\mathbf{1 2 4}$ & $\mathbf{2 3 . 2}$ \\
\hline$>61$ meses & 108 & 20.2 \\
\hline Total & 535 & 100 \\
\hline
\end{tabular}


Labrador et al. (2010) refiere en su trabajo el retraso en la consulta al psicólogo: habla de 4 años de espera previo a la consulta.

6) Motivo de la consulta

Tabla 6. Motivo de consulta.

\begin{tabular}{lll}
\hline Motivo de la consulta & Frecuencia & $\mathbf{\%}$ \\
\hline Adaptación & 88 & 16.4 \\
\hline Ansiedad & 94 & 17.6 \\
\hline Pareja & 87 & 16.3 \\
\hline Estado de ánimo & 98 & 18.3 \\
\hline Inseguridad personal & 65 & 15.3 \\
\hline Problemas relacionales & 43 & 8.0 \\
\hline Otros & 43 & 8.0 \\
\hline Total & 535 & 100 \\
\hline
\end{tabular}

Nuestros datos no se pueden comparar de manera precisa con los de otros estudios puesto que la categorización es distinta; en nuestro estudio diferenciamos entre motivo de consulta y diagnóstico clínico. Sin embargo, es coincidente el destacar problemas de adaptación y familiares (Sender 2001) o de adaptación, de estado de ánimo, de ansiedad, autoestima, y relaciones (Calero et al., 2018), o motivos familiares, sociales y de pareja (Quesada 2004) o de ansiedad-depresión, duelos, relaciones y adaptación (Salaberría et al., 2016), subjetivos o de relación (Gómez y Pérez, 2017).

7) Tratamiento psicofarmacológico

Tabla 7. Tratamiento psicofarmacológico.

\begin{tabular}{lll}
\hline Tratamiento farmacológico & Frecuencia & $\mathbf{\%}$ \\
\hline $\mathbf{S i}$ & $\mathbf{1 2 2}$ & $\mathbf{2 2 . 8}$ \\
\hline No & 413 & 77.2 \\
\hline total & 535 & 100 \\
\hline
\end{tabular}

Únicamente Salaberría et al. (2016) y Saldaña et al. (2009) refieren este concepto en sus estudios. Salaberría et al. (2016) encuentra que un $19.8 \%$ está en tratamiento psicofarmacológico, mientras Saldaña et al. (2009) habla de un $31.1 \%$. 
8) Diagnóstico

Tabla 8. Diagnóstico.

\begin{tabular}{lll}
\hline Diagnóstico & Frecuencia & $\mathbf{\%}$ \\
\hline T. Adaptación & 98 & 18.3 \\
\hline T. adaptativo depresivo & 72 & 13.5 \\
\hline T. adaptativo de ansiedad & 41 & 7.7 \\
\hline T. Ansiedad & 52 & 9.7 \\
\hline Crisis de ansiedad & 47 & 8.8 \\
\hline Fobias & 48 & 9.0 \\
\hline Duelos & 53 & 9.9 \\
\hline T. Depresivo & 48 & 9.0 \\
\hline Personalidad & 45 & 8.4 \\
\hline Otros & 31 & 5.8 \\
\hline Total & 535 & 100 \\
\hline
\end{tabular}

Nuestros hallazgos diagnósticos son bastante coincidentes con los del resto de trabajos sobre servicios universitarios en cuanto que destacan la baja tasa de diagnósticos de trastornos mentales más graves, excepción hecha del trabajo de Gutiérrez (2009) que encuentra un 10\% de psicosis. No obstante, las tasas halladas, no lo son; nuestra tasa de trastornos de ansiedad está próxima a la de Martorell y Carrasco (2009), García-Vera y Sanz (2009), Tejedo y López (2009), Labrador et al. (2010) y Avila et al. (2009); en trastorno adaptativo únicamente con nuestro trabajo de 2009; en trastorno de personalidad, con la de Martorell y Carrasco (2009); trastorno del estado de ánimo, con la de Gutiérrez (2009), Botella, Baños, GarcíaPalacios y Quero (2009) y Avila et al. (2009).

\section{A.2. Análisis de interacciones de variables}

Hemos encontrado que 32 interacciones se comportan como variables dependientes y 24 como variables independientes (ver tabla 9). 
Tabla 9. Interacciones de variables.

\begin{tabular}{lllllllll}
\hline & $P$ & $T$ & $M$ & $T R$ & $C$ & $E$ & $S$ & $D$ \\
\hline Primera $(P)$ & & $\mathrm{Si}$ & $\mathrm{Si}$ & $\mathrm{Si}$ & $\mathrm{No}$ & $\mathrm{Si}$ & $\mathrm{No}$ & $\mathrm{Si}$ \\
\hline Tiempo $(T)$ & $\mathrm{Si}$ & & $\mathrm{Si}$ & $\mathrm{No}$ & $\mathrm{No}$ & $\mathrm{Si}$ & $\mathrm{No}$ & $\mathrm{Si}$ \\
\hline Motivo $(M)$ & $\mathrm{Si}$ & $\mathrm{Si}$ & & $\mathrm{Si}$ & $\mathrm{Si}$ & $\mathrm{Si}$ & $\mathrm{No}$ & $\mathrm{Si}$ \\
\hline Tratamiento $(T R)$ & $\mathrm{Si}$ & $\mathrm{No}$ & $\mathrm{Si}$ & & $\mathrm{No}$ & $\mathrm{Si}$ & $\mathrm{No}$ & $\mathrm{Si}$ \\
\hline Colectivo $(C)$ & $\mathrm{No}$ & $\mathrm{No}$ & $\mathrm{Si}$ & $\mathrm{No}$ & & $\mathrm{Si}$ & $\mathrm{No}$ & $\mathrm{Si}$ \\
\hline Edad $(E)$ & $\mathrm{Si}$ & $\mathrm{Si}$ & $\mathrm{Si}$ & $\mathrm{Si}$ & $\mathrm{Si}$ & & $\mathrm{No}$ & $\mathrm{No}$ \\
\hline Sexo $(S)$ & $\mathrm{No}$ & $\mathrm{No}$ & $\mathrm{No}$ & $\mathrm{No}$ & $\mathrm{No}$ & $\mathrm{No}$ & & No \\
\hline Diagnóstico $(D)$ & $\mathrm{Si}$ & $\mathrm{Si}$ & $\mathrm{Si}$ & $\mathrm{Si}$ & $\mathrm{Si}$ & $\mathrm{No}$ & $\mathrm{No}$ & \\
\hline
\end{tabular}

Nota: Sí=dependiente, $\mathrm{No}=$ independiente.

De las 32 interacciones de variables que resultan dependientes, estadísticamente significativas, todas cumplen con los requisitos de la prueba de Chi Square. Nos planteamos explorar ¿qué pasa en torno a la realidad de las primeras consultas con el psicólogo? ¿Cómo llegan los pacientes a la consulta, en qué condiciones y qué les motiva a consultar? ¿Cómo está supeditado el diagnóstico por esas condiciones?

No podemos atender más que a aquellas variables que se han mostrado como variables dependientes. Así, no recurriremos a interpretar los datos de las variables "edad"y "sexo"porque se comportan como variables independientes respecto de la variable diagnóstico.

\section{Primera x Diagnóstico}

Los valores de la prueba de Chi Squared (37.134 (9), $p<0.001)$ muestran que las variables son dependientes ${ }^{7}$ (ver tabla 10$)$.

¿Condiciona el diagnóstico el que sea la primera vez que se consulta al psicólogo? Los datos de que disponemos informan claramente de que sí lo condicionan. ¿En qué sentido? La realidad de que sea la primera vez que se consulta promueve diagnósticos más adaptativos y de duelo que la contraria: si no es la primera vez que se consulta al psicólogo aparecen diagnósticos de trastornos de ansiedad, depresivos, de personalidad y otros. 
Tabla 10. Diagnóstico x Primera consulta.

\begin{tabular}{|c|c|c|c|}
\hline & $\begin{array}{c}\text { Sin visita previa } \\
(0)\end{array}$ & $\begin{array}{c}\text { Con visita previa } \\
(1)\end{array}$ & Total \\
\hline Adaptativo Casos & 23 & 75 & 98 \\
\hline Esperado & 29.492 & 68.508 & \\
\hline Adaptativo Ansiedad Casos & 8 & 33 & 41 \\
\hline Esperado & 12.338 & 28.662 & \\
\hline Adaptativo Depresivo Casos & 16 & 56 & 72 \\
\hline Esperado & 21.667 & 50.333 & \\
\hline Ansiedad Casos & 19 & 33 & 52 \\
\hline Esperado & 15.649 & 36.351 & \\
\hline Crisis Ansiedad Casos & 16 & 31 & 47 \\
\hline Esperado & 14.144 & 32.856 & \\
\hline Fobias Casos & 17 & 31 & 48 \\
\hline Esperado & 14.445 & 33.555 & \\
\hline Duelo Casos & 6 & 47 & 53 \\
\hline Esperado & 15.950 & 37.050 & \\
\hline T.Depresivo Casos & 28 & 20 & 48 \\
\hline Esperado & 14.445 & 33.555 & \\
\hline Personalidad Casos & 17 & 28 & 45 \\
\hline Esperado & 13.542 & 31.458 & \\
\hline Otros Casos & 11 & 20 & 31 \\
\hline Esperado & 9.329 & 21.671 & \\
\hline Total & 161 & 374 & 535 \\
\hline
\end{tabular}

Tiempo de aparición de la sintomatología x Diagnóstico

El resultado de la prueba de Chi Squared (121.87 (36), $p<0.001)$ muestra que las variables son dependientes.

Nos preguntamos si el tiempo de aparición de los síntomas condiciona el diagnóstico ¿Unos tiempos de síntomas promueven unos diagnósticos más que otros? (ver tabla 11). 
Tabla 11. Diagnóstico x Tiempo de aparición de la sintomatología.

\begin{tabular}{lcccccc}
\hline Diagnóstico & $<\mathbf{1}$ mes & $\begin{array}{c}\mathbf{2 - 4} \\
\text { meses }\end{array}$ & $\begin{array}{c}\mathbf{5 - 1 2} \\
\text { meses }\end{array}$ & $\begin{array}{c}\mathbf{1 3 - 6 0} \\
\text { meses }\end{array}$ & $\begin{array}{c}\text { (61 } \\
\text { meses }\end{array}$ & Total \\
\hline Adaptativo Casos & 10 & 21 & 25 & 27 & 15 & 98 \\
\hline Esperado & 10.441 & 22.348 & 22.714 & 22.714 & 19.783 & \\
\hline Adaptativo Ansiedad Casos & 6 & 15 & 12 & 6 & 2 & 41 \\
\hline Esperado & 4.368 & 9.350 & 9.503 & 9.503 & 8.277 & \\
\hline Adaptativo Depresivo Casos & 5 & $\mathbf{1 7}$ & 25 & 16 & 9 & 72 \\
\hline Esperado & 7.671 & 16.419 & 16.688 & 16.688 & 14.535 & \\
\hline Ansiedad Casos & 4 & 9 & 10 & 9 & 20 & 52 \\
\hline Esperado & 5.540 & 11.858 & 12.052 & 12.052 & 10.497 & \\
\hline Crisis Ansiedad Casos & 12 & 14 & 8 & 7 & 6 & 47 \\
\hline Esperado & 5.007 & 10.718 & 10.893 & 10.893 & 9.488 & \\
\hline Fobias Casos & $\mathbf{2}$ & 8 & 6 & 12 & 20 & 48 \\
\hline Esperado & 5.114 & 10.946 & 11.125 & 11.125 & 9.690 & \\
\hline Duelo Casos & 12 & 21 & 12 & 8 & 0 & 53 \\
\hline Esperado & 5.647 & 12.086 & 12.284 & 12.284 & 10.699 & \\
\hline T.Depresivo Casos & 4 & 9 & 13 & 15 & 7 & 48 \\
\hline Esperado & 5.114 & 10.946 & 11.125 & 11.125 & 9.690 & \\
\hline Personalidad Casos & 2 & 4 & 6 & 12 & 21 & 45 \\
\hline Esperado & 4.794 & 10.262 & 10.430 & 10.430 & 9.084 & \\
\hline Otros Casos & $\mathbf{0}$ & 4 & 7 & 12 & 8 & 31 \\
\hline Esperado & 7.069 & 7.185 & 7.185 & 6.258 & \\
\hline Total & 122 & 124 & 124 & 108 & 535 \\
\hline & 503 & & & & & \\
\hline
\end{tabular}

Si atendemos a los diagnósticos encontramos los siguientes datos significativos: los trastornos adaptativos en general, se presentan más tarde que los adaptativos de ansiedad o adaptativos depresivos, es decir, hasta 13-60 meses después de los primeros síntomas, mientras los trastornos adaptativos ansiosos consultan en intervalos más breves, $<1$ mes $\mathrm{y}<12$ meses; los trastornos adaptativos depresivos, mayoritariamente entre 5-12 meses.

Los trastornos ansiosos muestran una bivalencia en los tiempos de consulta: por un lado, las crisis de ansiedad consultan en intervalos menores de tiempo, $<4$ meses, en cambio, los trastornos fóbicos y el resto de trastornos de ansiedad $>13$ meses, sobre todo, $<61$ meses. Los duelos se presentan en intervalos $<4$ meses, mientras los trastornos claramente depresivos entre 5-60 meses. Los trastornos de personalidad y otros, se dan $>13$ meses.

Si atendemos a los intervalos de tiempo, resulta que $<1$ mes se dan los duelos, los trastornos adaptativos ansiosos y las crisis de ansiedad. Entre 2-4 meses, los 
trastornos adaptativos ansiosos y adaptativos depresivos, las crisis de ansiedad y los duelos. Entre 5-12 meses, los trastornos adaptativos sin especificar, los trastornos adaptativos ansiosos, los trastornos adaptativos depresivos y los trastornos depresivos. Entre 13-60 meses, otros trastornos, trastornos depresivos, adaptativos, de personalidad y fóbicos. $<61$ meses: Personalidad, fóbicos, ansiedad y otros.

\section{Tratamiento x Diagnóstico}

La prueba de Chi Squared (70.664 (9), $p<0.001$ ) muestra que las variables son dependientes. La cuestión se nos formula también respecto de la incidencia en el diagnóstico, ¿la realidad del tratamiento farmacológico favorece unos diagnósticos más que otros? (ver tabla 12).

Encontramos 5 categorías diagnósticos que no están en tratamiento psicofarmacológico en el momento de la consulta a la UAP, mientras otras 5 categorías sí lo están, si bien, las tasas de usuarios son mucho mayores en los que no están en tratamiento (más de las 3/4 partes). Vemos que los trastornos depresivos y las crisis de ansiedad están en tratamiento con datos bastante por encima de los esperados, los trastornos de ansiedad también, aunque menos, así como los de personalidad y otros, que los están de manera más discreta. 
Tabla 12. Diagnóstico x Tratamiento psicofarmacológico.

\begin{tabular}{lccc}
\hline Diagnóstico & $\begin{array}{c}\text { No tratamiento } \\
\text { previo(0) }\end{array}$ & $\begin{array}{c}\text { Sí tratamiento } \\
\text { previo(1) }\end{array}$ & Total \\
\hline Adaptativo Casos & 88 & 10 & 98 \\
\hline Esperado & 75.652 & 22.348 & \\
\hline Adaptativo Ansiedad Casos & 35 & 6 & 41 \\
\hline Esperado & 31.650 & 9.350 & \\
\hline Adaptativo depresivo Casos & 66 & $\mathbf{6}$ & 72 \\
\hline Esperado & 55.581 & 16.419 & \\
\hline Ansiedad Casos & 36 & 9 & 52 \\
\hline Esperado & 40.142 & 11.858 & \\
\hline Crisis Ansiedad Casos & 26 & 14 & 47 \\
\hline Esperado & 36.282 & 10.718 & \\
\hline Fobias Casos & $\mathbf{3 8}$ & 10 & 48 \\
\hline Esperado & 37.054 & 10.946 & \\
\hline Duelo Casos & 48 & 5 & 53 \\
\hline Esperado & 40.914 & 12.086 & \\
\hline T.Depresivo Casos & 21 & 27 & 48 \\
\hline Esperado & 27.054 & 10.946 & \\
\hline Personalidad Casos & 33 & 12 & 45 \\
\hline Esperado & 34.738 & 10.262 & \\
\hline Otros Casos & $\mathbf{2 2}$ & 9 & 31 \\
\hline Esperado & 23.931 & 7.069 & \\
\hline Total & 413 & 122 & 535 \\
\hline
\end{tabular}

El resto de diagnósticos, todos los trastornos adaptativos, los duelos y las fobias -más discretamente- no están en tratamiento psicofarmacológico.

\section{Colectivo x Diagnóstico}

Los valores de la prueba de Chi Squared (46.619 (27), $\mathrm{p}<0.011)$ muestran que las variables tienen una relación de dependencia. El colectivo iinfluye en el diagnóstico?, ¿unos colectivos tienen unos diagnósticos y otros colectivos otros? (ver tabla 13).

Encontramos unos diagnósticos más prevalentes que otros en unos colectivos que otros. El colectivo universitario muestra tasas más elevadas de trastornos de ansiedad, de fóbicos, de personalidad y de adaptativos depresivos. Los becarios, trastornos adaptativos depresivos, duelos y fobias. El PAS adaptativos ansiosos y adaptativos depresivos, crisis de ansiedad, duelos y depresivos. EL PDI trastornos 
adaptativos, duelos y otros. Siendo, por tanto, los trastornos adaptativos depresivos y los duelos las categorías más frecuentes en el conjunto de colectivos.

Tabla 13. Diagnóstico $\times$ Colectivo.

\begin{tabular}{lccccc}
\hline Diagnóstico & $\begin{array}{c}\text { Colectivo } \\
\text { Universitario }\end{array}$ & Becario & PAS & PDI & Total \\
\hline Adaptativo Casos & 61 & 6 & 18 & 13 & 98 \\
\hline Esperado & 65.028 & 6.778 & 19.050 & 7.144 & \\
\hline Adaptativo Ansiedad Casos & 20 & 2 & 16 & 3 & 41 \\
\hline Esperado & 27.206 & 2.836 & 7.970 & 2.989 & \\
\hline Adaptativo Depresivo Casos & 48 & $\mathbf{7}$ & 16 & 1 & 72 \\
\hline Esperado & 47.776 & 4.979 & 13.996 & 5.249 & \\
\hline Ansiedad Casos & 42 & 4 & 6 & 0 & 52 \\
\hline Esperado & 34.505 & 3.596 & 10.108 & 3.791 & \\
\hline Crisis ansiedad Casos & 34 & 1 & 11 & 1 & 47 \\
\hline Esperado & 31.187 & 3.250 & 9.136 & 3.426 & \\
\hline Fobias Casos & $\mathbf{3 6}$ & 5 & 3 & 4 & 48 \\
\hline Esperado & 31.850 & 3.320 & 9.331 & 3.499 & \\
\hline Duelo Casos & 29 & 5 & 11 & 8 & 53 \\
\hline Esperado & 35.168 & 3.665 & 10.303 & 3.864 & \\
\hline T.Depresivo Casos & 31 & 3 & 11 & 3 & 48 \\
\hline Esperado & 31.850 & 3.320 & 9.331 & 3.499 & \\
\hline Personalidad Casos & 34 & 2 & 7 & 2 & 45 \\
\hline Esperado & 29.860 & 3.112 & 8.748 & 6.280 & \\
\hline Otros Casos & 20 & 2 & 5 & 4 & 31 \\
\hline Esperado & 20.570 & 2.144 & 6.026 & 2.260 & \\
\hline Total & 355 & 37 & 124 & 39 & 535 \\
\hline
\end{tabular}

\section{Motivo x Diagnóstico}

La prueba de Chi Squared (600.384 (54), $p<0.001)$ muestra valores que permiten afirmar la dependencia de las dos variables. ¿Qué diagnósticos tienen nuestros usuarios que nos consultan en función del motivo de consulta? (ver tabla 14).

El trastorno de adaptación se presenta, con datos por encima de los esperados, en los motivos adaptación, inseguridad y pareja. El trastorno adaptativo depresivo en estado de ánimo y adaptación. El trastorno adaptativo ansioso en adaptación, ansiedad y relaciones. El trastorno de ansiedad en ansiedad, otros e inseguridad. Las crisis de ansiedad en ansiedad. Las fobias en ansiedad e inseguridad. Los duelos en 
pareja y estado de ánimo. El trastorno depresivo en estado de ánimo. El trastorno de personalidad en relaciones e inseguridad. Otros trastornos en otros e inseguridad.

Tabla 14. Diagnóstico x Motivo de consulta.

\begin{tabular}{lccccccccc}
\hline Diagnóstico & Adaptación & Ánimo & Ansiedad & Pareja & Relaciones & Inseguridad & Otros & Total \\
\hline Adaptativo Casos & 27 & 14 & 1 & 22 & 6 & 24 & 4 & 98 \\
\hline Esperado & 16.120 & 17.951 & 17.219 & 15.936 & 7.877 & 15.021 & 7.877 & \\
\hline Adaptativo & 15 & 0 & 12 & 2 & 5 & 6 & 1 & 41 \\
Ansiedad Casos & & & & & & & & \\
\hline Esperado & 6.744 & 7.510 & 7.204 & 6.667 & 3.295 & 6.284 & 3.295 & \\
\hline Adaptativo & 23 & 29 & 1 & 8 & 3 & 7 & 1 & 72 \\
Depresivo Casos & & & & & & & & \\
\hline Esperado & 11.843 & 13.189 & 12.650 & 11.708 & 5.787 & 11.036 & 5.787 & \\
\hline Ansiedad Casos & 4 & 2 & 23 & 2 & 2 & 9 & 10 & 52 \\
\hline Esperado & 8.553 & 9.525 & 9.136 & 8.456 & 4.179 & 7.970 & 4.179 & \\
\hline Crisis Ansiedad Casos & 1 & 2 & 34 & 4 & 4 & 1 & 1 & 47 \\
\hline Esperado & 7.731 & 8.609 & 8.258 & 7.643 & 3.778 & 7.204 & 3.778 & \\
\hline Fobias Casos & 7 & 4 & 20 & 2 & 3 & 9 & 3 & 48 \\
\hline Esperado & 7.895 & 8.793 & 8.434 & 7.806 & 3.858 & 7.357 & 3.858 & \\
\hline Duelo Casos & 0 & 13 & 1 & 38 & 0 & 0 & 1 & 53 \\
\hline Esperado & 8.718 & 9.708 & 9.312 & 8.619 & 4.260 & 8.123 & 4.260 & \\
\hline T.Depresivo Casos & 1 & 28 & 0 & 4 & 4 & 8 & 3 & 48 \\
\hline Esperado & 7.895 & 8.793 & 8.434 & 7.806 & 3.858 & 7.357 & 3.858 & \\
\hline Personalidad Casos & 7 & 4 & 1 & 3 & 15 & 12 & 3 & 45 \\
\hline Esperado & 7.402 & 8.243 & 7.907 & 7.318 & 3.617 & 6.897 & 3.617 & \\
\hline Otros Casos & $\mathbf{3}$ & 2 & 1 & 2 & 1 & 6 & 16 & 31 \\
\hline Esperado & 5.099 & 5.679 & 4.447 & 5.041 & 2.492 & 4.751 & 2.492 & \\
\hline Total & 88 & 98 & 94 & 87 & 43 & 82 & 43 & 535 \\
\hline
\end{tabular}

Si lo vemos desde la perspectiva de los motivos encontramos que el motivo de adaptación da lugar a diagnósticos de trastornos: adaptativos, adaptativos depresivos y adaptativos de ansiedad. El motivo de ansiedad a trastornos adaptativos de ansiedad, ansiedad, crisis de ansiedad y fobias. El motivo de pareja da lugar a trastornos adaptativos y duelo. Estado de ánimo a trastornos adaptativo depresivo, duelo y trastorno depresivo. Inseguridad personal a trastornos de adaptación, de ansiedad, fobias y otros. Problemas relacionales a trastornos de personalidad. Otros a trastornos de ansiedad y otros. 


\section{Discusión}

La descripción de la población estudiada presenta un perfil de edad media de casi 33 años, con una mediana de 30 años. El límite de edad inferior en 18 años es congruente con la edad mínima para acceder a la universidad; asimismo el límite superior en 67 sugiere el límite de la edad máxima laboral. Los percentiles 25 y 75, con edades de 25 años y 38 años, respectivamente, muestran que el grueso de la población atendida en el UAP es una población joven-adulta. La edad muestra una distribución de edades diferenciada respecto del colectivo, mientras las edades $<30$ años son más frecuentes en el colectivo de universitarios, $\operatorname{los}>31$ años se encuentran en el resto de colectivos (becarios, PAS y PDI). El PAS es el que presenta una distribución más dispersa de edades por encima de lo esperado.

El hecho de que el $67.3 \%$ de las personas atendidas sean mujeres también es coincidente con otros estudios en Salud Mental, así como con los de otros servicios universitarios de psicología. Por otro lado, que las $2 / 3$ partes de los consultantes sean estudiantes universitarios (66.4\%) muestra, quizás, el porqué de la juventud de la población. Cabe destacar igualmente que prácticamente en un $70 \%$ de los casos se trata de primeras consultas al profesional psicólogo clínico. Asimismo, más del $77 \%$ de los casos no está en tratamiento farmacológico en el momento de la consulta al UAP, frente al $22.8 \%$ que sí lo está; dato que se corrige un poco cuando se trata de la población $>51$ años, bajando al $63.33 \%$.

El tiempo que se demoran los consultantes para solicitar una sesión desde la aparición de los síntomas muestra que 1/3 de los mismos consultan antes de 4 meses. Sólo un $10.7 \%$ lo hace en un período de menos de un mes. Curiosamente el PAS es el colectivo que consulta en ese lapso de tiempo antes que otro; ¿será por la proximidad geográfica del lugar de trabajo? También es de destacar que un $43.4 \%$ espera más de un año para poder consultar al psicólogo. Los universitarios parecen dividirse en dos subgrupos, los que consultan antes de 4 meses y aquellos que lo hacen después de 5 años. El PDI muestra cifras de casi el 50\% consultando entre 1360 meses.

En cuanto a los motivos de consulta se presenta un panorama suficientemente disperso sin destacar de manera prevalente uno sobre otro con excepción de los problemas relacionales y otros. Por el contrario, los diagnósticos agrupan a más de los $2 / 3$ partes del total en torno a los trastornos adaptativos en general, adaptativos depresivos y de ansiedad, estando en un lugar muy inferior los diagnósticos claramente depresivos, de personalidad y otros diagnósticos (tóxicos, alimentación, psicosis, etc.), como encuentran García-Vera (2004) y Calero et al. (2018). Un $39.5 \%$ de los diagnósticos responden a trastornos adaptativos (si sumamos el duelo se convertiría en un $49.4 \%$, es decir, la mitad de los diagnósticos), un $27{ }^{\prime} 5 \%$ a trastornos de ansiedad (García-Vera, 2004, encuentra un 26\%). Datos que no son congruentes con los que se pueden encontrar en los dispositivos de Salud Mental de la red sanitaria pública.

Si atendemos a la interacción de las variables encontramos un primer dato muy 
significativo que hace referencia a la variable sexo y que informa de la ausencia de relación estadísticamente significativa con ninguna otra variable. Así, están al mismo nivel, en lo que hace a las consultas psicológicas, tanto hombres como mujeres; cosa que tampoco es congruente con los datos de otros estudios del ámbito de la Salud Mental (Montero et al., 2004). Sin negar la mayor tasa de consultas de mujeres, ya Sender (2001) subraya la mayor disposición de la mujer a solicitar ayuda psicológica, el hecho de esta falta de significación estadística de los datos en la interacción de las variables con el sexo, podría ser sugestivo de interpretación hipotética: ¿cabe pensar que a mayor nivel cultural, - ¿la universidad sería un exponente de cultura?- se difuminan las diferencias entre hombres y mujeres?

Con respecto a la edad, aunque sí muestra relación de dependencia con 5 variables sobre 8 , en relación con la variable diagnóstico está en relación de independencia, es decir, no condiciona el diagnóstico; no podemos afirmar que unas edades sean más propensas que otras a un diagnóstico antes que a otro. Sin embargo, sí hemos comprobado como cruzando las variables dependientes diagnóstico y motivo con la edad, sí se muestran como variables dependientes para todas las franjas de edad (cosa que no sucede cruzando la edad con las otras 4 interacciones de variables dependientes con el diagnóstico). ¿Por qué? Parecería que el sufrimiento psíquico se reparte por igual independientemente de la edad: no hay maneras de enfermar propias de las edades de nuestros usuarios.

Si focalizamos la atención en el hecho de que se trate de una primera visita obtenemos una distribución particular en el sentido de que la realidad de que existan o no consultas previas a la actual sí determina el diagnóstico. Si no las hay, y se trata de una primera visita, predominan los trastornos adaptativos y de duelo, lo cual informa de que en nuestra UAP se consulta por trastornos menos graves que comprometen un sufrimiento mental menor; cuando, por el contrario, sí que ha habido consultas previas (cerca de un 30\% de los usuarios) aparecen diagnósticos de trastornos de ansiedad, depresivos, de personalidad y otros, por tanto, trastornos mentales más consolidados. Para los que consultan por primera vez, quizás podemos hipotetizar que la cercanía al Campus, tanto para los estudiantes como para los trabajadores, y la gratuidad del servicio, así como la mayor disponibilidad del SAS respecto de las listas de espera de los equipamientos públicos de la red de Salud Mental, quizás promueven la decisión de consultar en la UAP. Posiblemente, el SAS puede ser visto como un lugar menos estigmatizado en "salud mental"que los de la red pública. Para los que ya han consultado previamente, tanto en la pública como en la privada, pueden valer estos condicionantes, igualmente, aunque los trastornos sean de otro nivel.

Respecto del tiempo de duración de la sintomatología también hemos podido comprobar que los tiempos son distintos para unos y otros diagnósticos. Así, a mayor tiempo de duración de la sintomatología ( $>61$ meses) encontramos ansiedad, fobias y personalidad de manera muy notoria, muy por encima de lo esperable; en menor grado, otros. Por el contrario, en el tiempo menor ( $<1$ mes, 2-4 meses), crisis 
de ansiedad, duelos y trastornos adaptativos ansiosos. En los intervalos intermedios (5-12 meses) encontramos los trastornos adaptativos, los adaptativos depresivos y los depresivos. Y en la franja intermedia mayor (13-60 meses) tanto los diagnósticos de uno como de otro polo: adaptativos, depresivos y otros; en menor grado fobias y de personalidad.

Es interesante observar como se diferencian los diagnósticos incluso dentro de una misma categoría diagnóstica, como por ejemplo la ansiedad: mientras las crisis de ansiedad no permiten espera, ni los adaptativos ansiosos, sí lo hacen las fobias o los trastornos de ansiedad en general. Debe ser la invasión del síntoma en la vida cotidiana la que permite menos espera que la que puede darse en una fobia que, por definición está limitada a la presencia del estímulo fobígeno, sea cual fuere. Por otro lado, los trastornos que comprometen el estado anímico, como los depresivos o los adaptativos con ánimo deprimido, pueden esperar más que los duelos. Probablemente, la invasión de las pérdidas que suponen los distintos duelos, mueven de manera más rápida a consultar que los estados anímicos depresivos que, por otro lado, sabemos que correlacionan en alto grado con consultas previas así como con tratamientos psicofarmacológicos por encima de lo esperado.

El primer dato significativo-ya lo hemos dicho-es que un $77 \%$ de los usuarios no están en tratamiento psicofarmacológico. El grueso de estos usuarios están diagnosticados como trastornos adaptativos, que representan el $89.57 \%$ de todos los diagnósticos por trastorno adaptativo en todas sus formas, trastornos fóbicos, el $79.17 \%$ de todos los diagnósticos fóbicos, y por duelos, el $90.56 \%$ de todos los diagnosticados. Estos diagnósticos suponen el $66.59 \%$ de los diagnósticos que no están en tratamiento, es decir, que aún encontraríamos, en menores porcentajes, otros diagnósticos que, aún no siendo estos diagnósticos prevalentes, tampoco están en tratamiento.

Si atendemos a los pacientes que sí están en tratamiento encontramos que el $56.25 \%$ (de los diagnósticos depresivos) padece trastorno depresivo, el 30.77\% (de los ansiosos) está diagnosticado por ansiedad, el 44.68\%, (de las crisis de ansiedad) por crisis de ansiedad, el 25\% (de los trastornos de personalidad) por trastorno de la personalidad y el $29 \%$ (de los otros) por otros diagnósticos. Es decir que estos diagnósticos condensan el $69.67 \%$ de todos los diagnósticos que están en tratamiento.

Cabe señalar como los pacientes que no están en tratamiento psicofarmacológico suman la mayoría de los trastornos adaptativos y duelos, en torno al $90 \%$, así como el $80 \%$ de las fobias. Buena noticia, sobre todo porque sabemos que esta dependencia de las variables (diagnóstico y tratamiento) se mantiene cruzándolas con la variable edad, con excepción de la franja de edad 41-50 años (que es el intervalo de edad de mayor proporción de PAS (57.14\%). Sabemos, por otro lado, que sólo el $21.88 \%$ de PAS de ese intervalo de años, está en tratamiento psicofarmacológico. Asimismo, el PDI de $>51$ años está en tratamiento el $50 \%$. Los universitarios y PAS de 31-40 años están en tratamiento en 22-23\%.

Entre los diagnósticos que sí están en tratamiento cabe señalar como aparece 
el trastorno depresivo en lugar prevalente, así como las crisis de ansiedad, junto a los otros diagnósticos (ansiedad, personalidad, otros). Este dato nos hace pensar que debe ser mayoritariamente el médico de atención primaria y, en menor grado, el psiquiatra, el que debe tratar estos casos, apareciendo así, una vez más, la preeminencia del recurso al tratamiento farmacológico por encima del psicológico pese a las indicaciones de las guías en Salud Mental, suponiendo como suponemos que estos diagnósticos no son de casos graves ${ }^{8}$.

En relación a los colectivos de la UAB también hemos encontrado datos específicos. En primer lugar, la prevalencia de los trastornos adaptativos depresivos y los duelos como las categorías más frecuentes en el conjunto de colectivos, si bien es verdad, los universitarios presentan duelos por debajo de lo esperado, así como los PDI presentan trastornos adaptativos depresivos, también muy por debajo de lo esperado. Los duelos están más presentes, proporcionalmente, en el PDI (20.51\%), menos en Becarios (13.51\%) y aún menos en PAS (10.58\%). Creemos que la edad debe estar influyendo en estos resultados, dado que el colectivo universitario es el más joven y el más numeroso; el PDI y el PAS el más mayor en edad. Probablemente los motivos del duelo no son los mismos, pero parece que la pareja y el estado de ánimo son los que hemos podido objetivar-como ya hemos visto. A mayor edad ¿más riesgo de estar expuesto a duelos? Tiene sentido.

En los trastornos adaptativos depresivos, elevados por encima de lo esperado en PAS y becarios, sugieren dificultades en torno al lugar de trabajo, pero quizás también en relación a las relaciones familiares y en general; nuestra experiencia clínica lo refiere. Los trastornos adaptativos en general, elevados en PDI, también preferimos interpretarlos como secundarios a tensiones laborales por encima de las dificultades extralaborales, por la misma razón. Por otro lado, la elevación de los datos de PAS en el trastorno adaptativo de ansiedad podemos interpretarla por razones laborales, en mayor grado, así como, en menor grado, por razones personales, familiares y otras.

Todos los trastornos de ansiedad, en sus diversas formas, se muestran elevados en los universitarios (34.93\% de todos los diagnósticos de universitarios), exclusivamente, es decir, en ningún otro colectivo lo están. Sabemos por la clínica que la situación académica de exigencia, así como en particular los exámenes, son una razón inductora de ansiedad, pero no la única; así, encontramos razones de índole diversa que pueden abonar la aparición de esos trastornos que ya hemos acreditado que no son, por otro lado, específicos de un colectivo. El colectivo PDI no muestra datos elevados en trastornos ansiosos, en ninguno. El PAS sí lo hace en crisis de ansiedad (10.58\%), mientras los Becarios en fobias (13.51\%). Datos curiosos, que no sabemos vincularlos a causas generales, propias de la condición del colectivo concreto.

El trastorno depresivo sólo aparece elevado, discretamente, en el colectivo PAS, aunque sólo representa el 10.58\% del total de diagnósticos, pero representando el $22.92 \%$ de todos los diagnósticos depresivos. La condición de PAS está más 
expuesta a este trastorno que la de otros colectivos, pero las razones no queremos atribuirlas al colectivo, exclusivamente, seguramente la edad está también detrás de esta exposición, ya que sabemos que la franja de edad 41-50, muy representativa en el PAS, es la única que mantiene la relación de dependencia de las variables diagnóstico y colectivo al cruzarla con la edad.

El trastorno de personalidad presenta unos datos muy claros: el $75.56 \%$ de los diagnósticos de ese trastorno son atribuidos a los universitarios, ¿por qué? Se nos ocurre que la menor edad del colectivo universitario puede influir en esta valoración diagnóstica; entre todos los diagnósticos universitarios representa el 9.58\% (frente al $6.73 \%$ de PAS; 5.41 de Becarios; y 5.13 de PDI), por tanto, no es que se trate de una categoría diagnóstica - una vez más- propia del colectivo universitario, ni mucho menos.

El motivo de consulta es la variable más rica y, por tanto, más compleja de interpretar; no olvidemos que supone 70 interacciones cruzar las dos variables, diagnóstico y motivo. Ciertamente que, si el motivo de consulta lo trae el paciente, el diagnóstico lo pone el profesional, pero teniendo en cuenta la realidad del paciente que, necesariamente, pasa por el motivo de la consulta. No se puede establecer una relación biunívoca entre motivo y diagnóstico, pero sí se muestran tendencias, una vez más.

El motivo "adaptación", mostrado como elevado por encima de lo esperable, sí que es exclusivo de todas las formas del trastorno adaptativo, es decir, no aparece en ninguna otra categoría diagnóstica, cosa que no sucede con ningún motivo más. Hay 88 motivos de adaptación y 65 en los diagnósticos de trastorno adaptativo, trastorno adaptativo ansioso y adaptativo depresivo, es decir, el 73.86\%. El motivo adaptación se encuentra también en trastornos de personalidad y fobias en una cifra muy próxima a la esperable, a diferencia del resto de trastornos que también lo presentan (ansiedad, crisis de ansiedad, depresivo y otros), pero muy por debajo. Sabemos que los trastornos adaptativos representan el 39.44\% del total, más de la tercera parte, siendo por tanto la categoría diagnóstica más alta; en muchos casos este motivo determina la valoración diagnóstica del clínico.

El motivo "estado de ánimo", como es lógico, aparece elevado por encima de lo esperado en los trastornos depresivos, el duelo y el trastorno adaptativo depresivo. E1 $71.43 \%$ de este motivo absorbe esos diagnósticos, considerados conjuntamente. No obtiene ningún registro en el trastorno adaptativo ansioso y sí en el resto de diagnósticos; particularmente próximo a la cifra esperable se encuentra el trastorno adaptativo. Aparece, pues, como un motivo claro que orienta el diagnóstico de manera bastante precisa.

El motivo "ansiedad"está presente en todos los diagnósticos de trastorno ansioso y en el adaptativo ansioso, lo que supone el $94.68 \%$ del total de esos diagnósticos juntos. Como vemos el motivo ansiedad correlaciona en alto grado con todos los diagnósticos que tienen ansiedad, lógicamente. Hemos confirmado como ningún motivo de ansiedad ha llevado a ningún diagnóstico de trastorno depresivo. 
Por el contrario, el motivo "estado de ánimo"sí lo hemos encontrado en 8 casos de trastornos ansiosos. Sólo en un caso aparece el motivo inseguridad en el diagnóstico de crisis de ansiedad y ninguno en duelo. Tampoco hay ningún registro ni del motivo "relaciones"ni “adaptación"en el duelo.

El motivo "pareja"elevado por encima de lo esperable en los trastornos adaptativo y duelo, supone el $62.07 \%$ de esos diagnósticos juntos. El motivo "pareja"no presenta registros próximos a los esperables en ningún diagnóstico, más allá de los indicados. Junto con el motivo "otros"tienen registros en todas las categorías diagnósticas, pero por debajo de lo esperable; son los únicos motivos que los tienen. Ambos diagnósticos sugieren que este motivo está representando problemáticas de relación de pareja que en gran medida suponen la ruptura del vínculo.

El motivo "relaciones"elevado por encima de lo esperable en los trastornos de personalidad y en los trastornos adaptativos ansiosos, supone el $46.51 \%$ de esos diagnósticos tomados conjuntamente. Este motivo también se manifiesta en cifras próximas a las esperables en el trastorno adaptativo, depresivo y crisis de ansiedad. Parece remitir a una problemática de sufrimiento más relacionada con la interacción con los otros, de manera especial los del clúster " $\mathrm{C}$ ", que es la categoría diagnóstica con mayores registros ${ }^{9}$. El clúster "A"y "B"juntos, sólo presentan 9 registros, frente a los 45 del total, es decir, la quinta parte ${ }^{10}$.

El motivo "inseguridad", también elevado por encima de lo esperable en los trastornos adaptativo, de ansiedad, fóbicos, de personalidad y otros, es el motivo más disperso, y supone el $73.9 \%$ de esos diagnósticos. También se encuentra levemente por encima de lo esperable en el trastorno depresivo y próximo a lo esperable en el trastorno adaptativo ansioso. Parece lógico esta dispersión del motivo entre tantos diagnósticos por cuanto la inseguridad (y la insatisfacción personal) son categorías suficientemente indefinidas susceptibles de hallarse en muchos diagnósticos.

El motivo "otros"está elevado en los trastornos de ansiedad y otros y representa el $60.47 \%$ del total de esos diagnósticos juntos. El motivo "otros"-que está presente en todos los diagnósticos- se encuentra en cifras próximas a las esperables en los trastornos fóbicos, de personalidad y depresivo. Tiene sentido también esta disparidad puesto que es una categoría dispersa que engloba problemas diversos (sexuales, laborales, de comportamiento, de sueño, etc..).

Nos hemos preguntado hasta qué punto las otras variables actuando como tercera variable modulan la interacción diagnóstico-motivo y hemos encontrado datos contundentes: en todos los casos se mantiene la dependencia de las variables, incluso cuando cruzamos con sexo y edad, con la excepción de la variable colectivo, concretamente, en el caso de los becarios, en quienes las variables diagnóstico y motivo se comportarían como independientes. 


\section{Conclusiones}

La población que consulta en la UAP de la UAB es una población adulta joven en comparación con otros dispositivos de la red pública de Salud Mental, a la que le cuesta consultar al psicólogo; necesita darse un tiempo antes de decidir consultar. Sólo un tercio de los consultantes lo hace dentro de los primeros 4 meses de aparición de la sintomatología. Si bien es verdad que cuanto mayor tiempo pasa teniendo síntomas más probabilidad hay de que se haya consultado previamente en otros dispositivos públicos o privados. En este sentido hemos encontrado una proporción muy superior de primeras consultas frente a las que no lo son: el 69.9\% es la primera vez que consulta al psicólogo clínico. Dato que es suficientemente significativo teniendo en cuenta la edad media de los consultantes, cerca de los 33 años.

Los diagnósticos clínicos ponen de manifiesto un espectro suficientemente disperso que se focaliza en unos u otros motivos de consulta y en función de otras características, como el tiempo de la sintomatología, el diagnóstico, la realidad de la primera consulta, o la existencia de tratamiento psicofarmacológico previo. La alta correlación entre el motivo de consulta y los diagnósticos desvela la alta tasa de motivos que tienen que ver con las reacciones agudas más o menos desadaptativas hasta el punto de que casi un $40 \%$ de los casos son orientados como trastornos adaptativos (un $49.35 \%$ si incluimos los duelos), frente al $27.5 \%$ de trastornos de ansiedad y el $9 \%$ de trastornos depresivos. Está claro que en nuestra UAP la tasa de los denominados trastornos mentales graves es prácticamente nula.

Igualmente, la tasa de los tratamientos psicofarmacológicos está lejos de ser la que se encuentra en las unidades de Salud Mental de la red pública. No llega a la cuarta parte la tasa de los que están medicados en el momento de la realización de la primera consulta; si focalizamos la atención en cada categoría diagnóstica encontramos que sólo el $10.43 \%$ de los trastornos adaptivos lo está, el $32 \%$ de los trastornos de ansiedad, el $56.25 \%$ de los trastornos depresivos, el $26.67 \%$ de los trastornos de personalidad y el $29 \%$ de otros trastornos. En este trabajo no podemos discriminar la gravedad de estos trastornos que tienen una tasa más alta de medicación psicofarmacológica.

\section{Notas}

1 Traducción al castellano: "Viernes Clínicos".

2 Desde 1995 a 2001 sí ofrecía una atención mixta, de hecho la Unidad se llamaba "Unitat d'Atenció Psicològica i psicopedagògica" pero partir de 2001 se escinde y se crea la "Unitat d'Atenció Psicopedagògica" que ofrece el abordaje psicopedagógico y la "Unitat d'Atenció Psicològica" ofrece la asistencia psicológica. http://gestioneventos.us.es/17468/programme/xv-encuentroaesppu.html

3 La Asociación Española de Servicios Psicológicos y Psicopedagógicos (AESPPU) creada en 2009 y que aglutina a una treintena de servicios universitarios ya ha realizado el XV Encuentro, concretamente en la Universidad de Sevilla, en Junio de 2018.

4 Los psicólogos especialistas en Psicología Clínica hacen el 82.5\% de la jornada. El resto, los psicólogos generales sanitarios. 
5 A este efecto cabe especificar que en nuestra UAP, un porcentaje de aproximadamente una tercera parte del volumen total de consultas en la UAP, ha requerido, durante los años a los que se refiere este trabajo, de una primera entrevista de psiquiatría para ser atendido por los profesionales psicólogos por prescripción del Seguro escolar, a pesar de que la demanda directa del usuario es de recibir atención psicológica.

6 Si elvalor dep es $<0.05$, las variables son dependientes entre sí; si el valor $\mathrm{p}$ es $>0.05$, las variables son independientes entre sí.

7 Los valores de chi squared están consignados en el primer número del paréntesis, seguidos de los grados de libertad -entreparèntesis- $y$ finalmente el valor de $\mathrm{p}$.

8 Las guías más valoradas son las de la Canadian Psychiatric Association, las del National Institute for Health and Clinical Excellence (NICE) y la National Collaborating Centre for Mental Health, de la British Psychological Society. En España existen las publicadas por el Ministerio de Sanidad y Consumo: Guías de práctica clínica en el SNS.

9 Clúster C: trastornos de personalidad evitativa, dependiente y obsesivo-compulsiva.

10 Clúster A: trastorno de personalidad esquizoide, esquizotípico y paranoide. Clúster B: Trastorno de personalidad antisocial, límite, histriónico y narcisista.

\section{Referencias bibliográficas}

American Psychiatric Association (2000). Manual Diagnóstico y Estadístico de los Trastornos Mentales (4. ${ }^{a}$ Ed.). Barcelona, España: Masson

Avila, A., Herrero Sánchez, J. R. y Fernández, L. C. (2009). Más allá de la docencia: la Unidad de Atención Psicológica y Salud Mental del Universitario (UAPSMU) en la Universidad de Salamanca (1996-2004). Acción Psicológica, 6(1), 49-62. https://doi.org/10.5944/ap.6.1.443

Botella, C., Baños, R. M., García-Palacios, A. y Quero, A. (2009). El servicio de asistencia psicológica de la Universitat Jaume I. Acción Psicológica, 6(1), 87-96. https://doi.org/10.5944/ap.6.1.447

Calero, E. A., Torre Martí, M. D. L., Pardo, R. y Santacreu, I. M. (2018). Características de la asistencia psicológica en el contexto universitario: la Clínica Universitaria de la UAM. Análisis y Modificación de Conducta, 44(169-170), 37-50. Recuperado de: https://dialnet.unirioja.es/servlet/articulo?codigo=6463438

García-Vera, M. P. (2004). Los centros universitarios de asistencia psicológica como centros de formación de postgrado en psicología clínica y psicoterapia. La unidad de psicología clínica y de la salud de la UCM. Papeles del psicólogo, 25(87), 59-66.

García-Vera, M. P. y Sanz, J. (2009). La clínica universitaria de psicología de la Universidad Complutense de Madrid: historia, características y situación actual. Acción Psicológica, 6(1), 123-134. Recuperado de: https://doi.org/10.5944/ap.6.1.451

Gómez, M. y Pérez, E. (2017). Particularidades de los motivos de consulta psicológica de los estudiantes que asisten a la Dirección de Bienestar Universitario, Universidad de Antioquia. Revista de Psicología Universidad de Antioquia, 9(1), 75-92. http://doi.org/10.17533/udea.rpsua.v9n1a06

Grupo de Trabajo de la Guía de Práctica Clínica para el Manejo de Pacientes con Trastornos de Ansiedad en Atención Priomaria (2008). Guia de Práctica Clínica para el Manejo de Pacientes con Trastornos de Ansiedad en Atención Primaria. (447-08-020-7). Madrid: Agencia Lain Entralgo. Recuperado de: http:// www.guiasalud.es/GPC/GPC_430_Ansiedad_Lain_Entr_compl.pdf

Grupo de trabajo de la Guía de Práctica Clínica sobre el Manejo de la Depresión en el Adulto (2014). Guía de Práctica Clínica sobre el Manejo de la Depresión en el adulto (680-14-079-0). Madrid: Agencia de Evaluación de Tecnologías Saniatarias de Galicia. Recuperado de: https://bit.ly/1VgMS86

Gutiérrez, E. (2009). La Unidad Venres Clínicos en la Universidad de Santiago de Compostela. Acción Psicológica, 6(1), 63-71. https://doi.org/10.5944/ap.6.1.444

Hernández, J. M. y Froján, M. X. (1994). El servicio de psicología aplicada de la UAM. Una experiencia de integración de la psicología en un contexto universitario. Papeles del Psicólogo, 1994, 58. Recuperado de: http://www.papelesdelpsicologo.es/resumen?pii=619

Labrador, F. J, Estupiñá, F. J. y García-Vera, M. P. (2010). Demanda de atención psicológica en la práctica clínica: tratamientos y resultados. Psicothema, 22(4), 619-626. Recuperado de: https://bit.ly/2JeEMw7 
Labrador, F. J., Bernaldo-de-Quirós, M., García-Fernández, G., Estupiñá, F. J., Fernández-Arias, I. y LabradorMéndez (2016). Characteristics of demand and psychological treatments in a university clinic [Características de la demanda y de los tratamientos psicológicos en una clínica universitaria]. Clínica y Salud, 27, 714. https://doi.org/10.1016/j.clysa.2016.01.002

Martorell, J. L. y Carrasco, M. A. (2009). La atención psicológica desde la Universidad Nacional de Educación a Distancia (UNED). El Servicio de Psicología Aplicada (SPA). Acción Psicológica, 6(1), 135-143. https://doi.org/10.5944/ap.6.1.452

Montero, I. Aparicio, D., Gómez-Beneyto, M., Moreno-Küstner, B., Reneses, B., Usall, J., Vázquez-Barquero, J. L. (2004). Género y salud mental en un mundo cambiante. Gaceta Sanitaria, 18(1), 175-181. Recuperado de: http://scielo.isciii.es/scielo.php?script=sci_arttext\&pid=S0213-91112004000400028

Narváez, My Aguirre, H. (2016). Análisis de las impresiones diagnósticas en consultantes de un Centro de Atención Psicológica Universitario. Informes Psicológicos, 16 (2), 87-101. https://bit.ly/2FTFz3M

Quesada, S. (2004). Estudio sobre los motivos de consulta psicológica en una población universitaria. Universitas Psychologica, 3(1), 7-16. https://doi.org/10.17533/udea.rpsua.v9n1a06

Salaberría, K., Polo-López, Ruiz-Iriondo, M., Cruz-Sáez, S. y Echeburúa, E. (2016). Análisis de la demanda en una unidad universitaria de asistencia psicológica. Revista Argentina de Clínica Psicológica, 25(3), 299-308.

Saldaña, C., Badós, A., García-Grau, E., Balaguer, G. y Fusté-Ecolano, A. (2009). La Unidad de Terapia de Conducta de la Universidad de Barcelona. Acción Psicológica, 6(1), 113-122. https://bit.ly/2S35VFi

Sánchez, M. F., Guillamón, J. R., Ferrer, P., Villalba, E., Martín, A. M. y Pérez, J. C. (2008). Situación actual de los Servicios de orientación universitaria: estudio descriptivo. Revista de educación, 345, 329-352. Recuperado de: http://www.revistaeducacion.mec.es/re345/re345_14.pdf

Santiago, B. (2009). La asesoría psicológica y social de la Universidad de Sevilla. Acción Psicológica, 6(1), $107-$ 111. https://doi.org/10.5944/ap.6.1.449

Saúl, L. A., López-González, M. A. y Bermejo, B. G. (2009). Revisión de los servicios de atención psicológica y psicopedagógica en las universidades españolas. Acción Psicológica, 6(1), 17-40. https://bit.ly/2LBGGZm

Sender, R. y Salamero, M. (2001). El Programa de Atención Psicológica de la Facultad de Medicina de la Universidad de Barcelona. Educación Médica, 4(2), 66-71. Recuperado de: https://bit.ly/2JqaJka

Tejedo, A. (2018, febrero 27). Día de la Psicología en España. La atención psicológica en la enseñanza pública universitaria en España. Psi Ara. Recuperado de: http://www.psiara.cat/view_article.asp?id=4804

Tejedo, A. y López, C. (2009). Presentación y descripción de la Unidad de Psicología del Servei Assistencial de Salut de la UAB. Acción Psicológica, 6(1), 97-105. https://doi.org/10.5944/ap.6.1.448 